\title{
A NEW CONSTRUCTION OF EINSTEIN SELF-DUAL METRICS*
}

\author{
RADU PANTILIE ${ }^{\dagger}$ AND JOHN C. WOOD $\ddagger$
}

\begin{abstract}
We give a new construction of Ricci-flat self-dual metrics which is a natural extension of the Gibbons-Hawking ansatz. We also give characterisations of both these constructions, and explain how they come from harmonic morphisms.
\end{abstract}

Introduction. In $[15,18]$, G.W. Gibbons and S.W. Hawking introduced a construction of Einstein self-dual metrics with zero scalar curvature (see [24] for a thorough discussion of this ansatz). The construction is in the spirit of Kaluza-Klein theory with the projection of the (local) bundle being a Riemannian submersion followed by a conformal transformation.

A harmonic morphism is a map between Riemannian manifolds which preserves Laplace's equation (see Section 2 below). In [7], R.L. Bryant gave a local normal form for the metric on the domain of a submersive harmonic morphism with one-dimensional fibres. When the domain is four-dimensional, this local normal form includes that of the Gibbons-Hawking ansatz.

In $[26,27]$, it is shown that, from an Einstein four-manifold, there are precisely three types of harmonic morphism with one-dimensional fibres. The first two types are due to R.L. Bryant and to P. Baird and J. Eells, respectively, and lead to the Gibbons-Hawking construction and the well-known warped product construction of Einstein metrics (see Theorem 1.1 and Theorem 1.2 below). The third type can be seen as a construction of Ricci-flat self-dual metrics. We present this new construction in Section 1 (Theorem 1.3) together with the above mentioned result from [26, 27] reformulated as a classification result for Einstein four-manifolds whose metric can be written in Bryant's local normal form (Theorem 1.5). In Section 2 we review some facts on harmonic morphisms. In Section 3 we give proofs of the results of Section 1 and show that our construction is a natural extension of the Gibbons-Hawking ansatz; indeed all three constructions are characterised by an equation (3.1) which generalizes the monopole equation. We also classify the harmonic morphisms with one-dimensional fibres on compact Einstein four-manifolds. Our new construction involves solving equation (1.5) below, which is a particular case of the Beltrami fields equation of hydrodynamics (see [20]). In Section 4 we describe all solutions of (1.5), both locally and globally, on $S^{3}$ by a method similar to the one used in [20] to describe solutions of the Beltrami fields equation on $\mathbb{R}^{3}$ (see Remark 4.2), giving all Einstein metrics of the form (1.4) below; in fact we show that for such metrics the Einstein condition is equivalent to the Beltrami fields equation.

We are very grateful to G.W. Gibbons and N.J. Hitchin for pointing out to us that (1.5) is a particular case of the Beltrami fields equation and for recommending references on this subject. We thank D.M.J. Calderbank for drawing our attention to his paper [8], see below, and F. Belgun for useful comments.

\footnotetext{
*Received February 22, 2002; accepted for publication March 5, 2002.

${ }^{\dagger}$ Deparment of Pure Mathematics, University of Leeds, Leeds LS2 9JT, G.B. (r.pantilie@leeds.ac.uk).

$\ddagger$ Deparment of Pure Mathematics, University of Leeds, Leeds LS2 9JT, G.B. (j.c.wood@leeds.ac.uk). The authors gratefully acknowledge that this work was done under E.P.S.R.C. grant number GR/N27897.
} 
1. The construction. Firstly, we recall the following ansatz of G.W. Gibbons and S.W. Hawking [15, 18] (see also, [24]).

THEOREM 1.1. Let $U \subseteq\left(\mathbb{R}^{3}, h\right)$ be an open subset of the Euclidean three-space. Let $u$ be a positive smooth function and $A$ a one-form on $U$. Then, the Riemannian metric on $\mathbb{R} \times U$ given by

$$
g=u h+u^{-1}(\mathrm{~d} t+A)^{2} \quad(t \in \mathbb{R})
$$

is Einstein if and only if $u$ and $A$ are related by the monopole equation:

$$
\mathrm{d} u=* \mathrm{~d} A
$$

with respect to a suitable choice of orientation on $U$. Moreover, if (1.2) holds, then $g$ is Ricci-flat and self-dual.

Note that (1.2) implies that $u$ is a harmonic function. Moreover, if, for example, $U$ is an open ball then, given a harmonic function $u$, we can find a one-form $A$ such that (1.2) is satisfied. Also, note that $A$ defines a principal connection on $(\mathbb{R} \times U, U, \mathbb{R})$ with curvature form $F=\mathrm{d} A$ which is harmonic, i.e., $\mathrm{d} F=0, \mathrm{~d}^{*} F=0$. Conversely, given a harmonic two-form $F$ on, say; an open ball $U$ of $\mathbb{R}^{3}$, solving $\mathrm{d} A=F$ and $\mathrm{d} u=* F$ gives a solution to (1.2).

Another way of obtaining Einstein metrics, to which our construction is related, is given by the following well-known warped product construction (see [5, 9.109]).

THEOREM 1.2. Let $\left(N^{n}, h\right)$ be an Einstein manifold of dimension $n$ with ${ }^{N_{\text {Ricci }}}=$ $c^{N} h$. Let $g$ be the warped product metric on $M^{n+1}=\mathbb{R} \times N^{n}$ given by $g=\mathrm{d} t^{2}+\lambda^{-2} h$ where $\lambda: \mathbb{R} \rightarrow(0, \infty)$ is a smooth function.

Then, $\left(M^{n+1}, g\right)$ is Einstein, with ${ }^{M}$ Ricci $=c^{M} g$, if and only if $\lambda$ satisfies the following equation:

$$
\frac{c^{M}}{n} \lambda^{2}-\frac{c^{N}}{n-1} \lambda^{4}+\left(\lambda^{\prime}\right)^{2}=0
$$

Moreover, if $\left(N^{n}, h\right)$ has constant curvature (note that this is automatically satisfied if $\operatorname{dim} N=3)$, then $\left(M^{n+1}, g\right)$ also has constant curvature.

Next, we give the new construction based on the Beltrami fields equation, see Proposition 4.1 for solutions.

THEOREM 1.3. Let $U \subseteq\left(S^{3}, h\right)$ be an open subset of the three-sphere endowed with its canonical metric.

Let $A$ be a one-form on $U$. Then, the Riemannian metric on $(0, \infty) \times U$ given by

$$
g=\rho^{2} h+\rho^{-2}(\rho \mathrm{d} \rho+A)^{2} \quad(\rho \in(0, \infty))
$$

is Einstein if and only if the following Beltrami fields equation holds on $U$ :

$$
\mathrm{d} A+2 * A=0
$$

with respect to a suitable choice of orientation on $U$. Moreover, if (1.5) holds, then $g$ is Ricci-flat and self-dual.

Remark 1.4. D.M.J. Calderbank [8, Theorem V] reduces the problem of finding scalar flat Kähler self-dual 4-manifolds to certain affine monopole equations on an 
Einstein-Weyl 3-manifold. For suitable choice of gauge, these equations reduce to the Beltrami fields equation.

The proof of Theorem 1.3 will be given in Section 3 .

By a local principal bundle $\left(M^{n+1}, N^{n}, S^{1}\right)$ (with principal connection $\mathcal{H} \subseteq T M$ ) we shall mean the restriction of a principal bundle $\left(\widetilde{M}^{n+1}, \widetilde{N}^{n}, S^{1}\right)$ (with principal connection $\widetilde{\mathcal{H}} \subseteq T \widetilde{M}$ ) to an open subset $M^{n+1}$ of $\widetilde{M}^{n+1}$. Then, the above three constructions admit the following characterisation which will be proved in Section 3.

Theorem 1.5. Let $\left(N^{3}, h\right)$ be a Riemannian three-manifold and let $\left(M^{4}, N^{3}, S^{1}\right)$ be a local principal bundle endowed with a principal connection $\mathcal{H} \subseteq T M$. Define a Riemannian metric $g$ on $M^{4}$ by

$$
g=\lambda^{-2} \varphi^{*}(h)+\lambda^{2} \theta^{2}
$$

where $\lambda$ is a positive smooth function on $M^{4}, \varphi: M^{4} \rightarrow N^{3}$ is the projection of the local principal bundle $\left(M^{4}, N^{3}, S^{1}\right)$ and $\theta$ is the connection form of $\mathcal{H}$.

Suppose that $\left(M^{4}, g\right)$ is Einstein.

(a) Then one of the following assertions holds:

(i) $S^{1}$ acts by isometries on $M^{4}$ (in particular, the fibres of $\varphi$ form a Riemannian foliation);

(ii) $\left(M^{4}, g\right)$ and $\left(N^{3}, h\right)$ have constant sectional curvature and, locally, $g$ is given by Theorem 1.2, with $n=3$;

(iii) $\left(N^{3}, h\right)$ has positive constant sectional curvature and, up to homotheties, $g$ is locally given by Theorem 1.3 .

(b) Furthermore, if $\left(N^{3}, h\right)$ has constant curvature then (i) can be replaced by

$\left(\mathrm{i}^{\prime}\right)\left(N^{3}, h\right)$ is flat and $g$ is locally given by Theorem 1.1 .

Thus if $\left(N^{3}, h\right)$ has constant curvature, the ansatz (1.6) gives an Einstein metric if and only if it is one of the three constructions: Gibbons-Hawking (Theorem 1.1), warped product (Theorem 1.2) or Beltrami fields (Theorem 1.3).

REMARK 1.6.

1) If both (i) and (ii) of Theorem 1.5 occur then, locally, $\left(M^{4}, g\right)$ is the Riemannian product $\mathbb{R} \times\left(N^{3}, h\right)$; whilst, if both (ii) and (iii) occur, then, locally and up to homotheties, $\left(N^{3}, h\right)$ is the three-sphere, $\left(M^{4}, g\right)$ is the Euclidean four-space and (1.6) corresponds to the polar coordinates. However, (i) and (iii) cannot occur simultaneously.

2) Note that a map $\varphi:\left(M^{n+1}, g\right) \rightarrow\left(N^{n}, h\right)$ is a submersive harmonic morphism if and only if it is locally as described in the hypothesis of Theorem 1.5 (see Theorem 2.6 below).

3) A similar result ([30]) can be given for a Riemannian manifold $N$ of any dimension $n \geq 4$. In that case, $g=\lambda^{-2} \varphi^{*}(h)+\lambda^{2 n-4} \theta^{2}$ (cf. [7]), and $g$ can be as in (1.4) only in the trivial case $A=0$.

4) Assertion (i) of Theorem 1.5 is equivalent to the assertion that $\lambda$ be constant along the fibres of $\varphi$, whereas, in case (ii), $\lambda$ is constant along horizontal curves (i.e., tangent to $\mathcal{H}$ ), see, also, Proposition 2.4 and Proposition 2.5, below.

5) In assertion (iii) of Theorem 1.5, we have $\rho=\lambda^{-1}$ where $\rho$ is as in Theorem 1.3. Similarly, in assertion $\left(i^{\prime}\right)$ of Theorem 1.5, we have $u=\lambda^{-2}$ where $u$ is as in Theorem 1.1 .

2. Some facts on harmonic morphisms. Both Theorem 1.3 and Theorem 1.5 have their origin in the theory of harmonic morphisms; in this section, we recall some 
basic facts from that theory (see [4] for a general account and [17] for a frequently updated bibliography). For simplicity, from now on all the manifolds are assumed to be smooth, connected and orientable.

Definition 2.1. A harmonic morphism is a smooth map $\varphi:\left(M^{m}, g\right) \rightarrow\left(N^{n}, h\right)$ between Riemannian manifolds which pulls back harmonic functions to harmonic functions, i.e., for any harmonic function $f:\left(U,\left.h\right|_{h}\right) \rightarrow \mathbb{R}$ defined on an open subset $U$ of $N^{n}$, with $\varphi^{-1}(U)$ non-empty, $f \circ \varphi:\left(\varphi^{-1}(U),\left.g\right|_{\varphi^{-1}(U)}\right) \rightarrow \mathbb{R}$ is a harmonic function.

To state the basic characterisation result for harmonic morphisms we also need the following.

Definition 2.2. A smooth map $\varphi:\left(M^{m}, g\right) \rightarrow\left(N^{n}, h\right)$ between Riemannian manifolds is horizontally (weakly) conformal if, at each point $x \in M$, either $\mathrm{d} \varphi_{x}=0$, or $\mathrm{d} \varphi_{x}: T_{x} M \rightarrow T_{\varphi(x)} N$ is surjective and its restriction to the horizontal space $\mathcal{H}_{x}=\left(\operatorname{ker} \mathrm{d} \varphi_{x}\right)^{\perp}$ is a conformal (linear) map $\left(\mathcal{H}_{x},\left.g_{x}\right|_{\mathcal{H}_{x}}\right) \rightarrow\left(T_{\varphi(x)} N, h_{\varphi(x)}\right)$. Denote the conformality factor by $\lambda(x)$. The resulting function $\lambda$ is called the dilation of $\varphi$. The dilation is smooth outside the set of critical points and can be extended to a continuous function on $M^{m}$, with $\lambda^{2}$ smooth, by setting it equal to zero on the set of critical points.

A smooth map is called horizontally homothetic if it is horizontally conformal with dilation constant along horizontal curves.

The following theorem is due to B. Fuglede [13] and T. Ishihara [19] .

THEOREM 2.3. A smooth map between Riemannian manifolds is a harmonic morphism if and only if it is a harmonic map which is horizontally weakly conformal.

Any Riemannian submersion with minimal fibres is a harmonic morphism. This can be seen either directly or by applying Theorem 2.3. Other simple examples are radial projection $\mathbb{R}^{n+1} \backslash\{0\} \rightarrow S^{n}(n \geq 1)$ defined by $x \mapsto x /|x|$ and the Hopf polynomial $\mathbb{C}^{2} \rightarrow \mathbb{R}^{3}$ defined by $\left(z_{1}, z_{2}\right) \mapsto\left(\left|z_{1}\right|^{2}-\left|z_{2}\right|^{2}, 2 z_{1} \overline{z_{2}}\right)$. Note that radial projection is a horizontally homothetic submersion with geodesic fibres, whilst the fibres of the Hopf polynomial are tangent to a Killing vector field. Both of these examples are particular cases of more general constructions which we now present.

Proposition $2.4([2])$. Let $\varphi:\left(M^{m}, g\right) \rightarrow\left(N^{n}, h\right)$ be a non-constant horizontally weakly conformal map. If $\operatorname{dim} N=2$, then $\varphi$ is a harmonic morphism if and only if its fibres are minimal at regular points. If $\operatorname{dim} N \neq 2$, then any two of the following assertions imply the third:

(i) $\varphi$ is a harmonic morphism;

(ii) the fibres of $\varphi$ are minimal at regular points;

(iii) $\varphi$ is horizontally homothetic.

From Proposition 2.4 it follows that any horizontally homothetic submersion with minimal fibres is a harmonic morphism.

Proposition 2.5 ([7]). For $n \geq 3$, let $\varphi:\left(M^{n+1}, g\right) \rightarrow N^{n}$ be a surjective submersion with one-dimensional fibres which form an orientable Riemannian foliation. Then the following assertions are equivalent:

(i) there exists a Riemannian metric $h$ on $N$ with respect to which the map $\varphi:\left(M^{n+1}, g\right) \rightarrow\left(N^{n}, h\right)$ is a harmonic morphism;

(ii) there exists a non-zero Killing vector field tangent to the fibres of $\varphi$.

From [7], we also recall the following characterisation.

THEOREM 2.6. Let $\left(M^{n+1}, N^{n}, S^{1}\right)$ be a principal bundle with projection $\varphi$ : $M^{n+1} \rightarrow N^{n}$ and endowed with a principal connection $\mathcal{H} \subseteq T M$. Let $h$ be a Rieman- 
nian metric on $N^{n}$ and $\lambda$ a smooth positive function on $M^{n+1}$.

Define a Riemannian metric on $M^{n+1}$ by

$$
g=\lambda^{-2} \varphi^{*}(h)+\lambda^{2 n-4} \theta^{2}
$$

where $\theta$ is the connection form of $\mathcal{H}$. Then $\varphi:\left(M^{n+1}, g\right) \rightarrow\left(N^{n}, h\right)$ is a harmonic morphism.

Conversely, any submersive harmonic morphism with one-dimensional fibres is locally of this form, up to isometries.

See [25], [26] for a proof of Theorem 2.6 and a more explicit version of the converse.

\section{REMARK 2.7.}

1) In the notations of Theorem 2.6, let $V$ be the vertical vector field with $\theta(V)=$ 1. Obviously, $V$ is the infinitesimal generator of the $S^{1}$ action and $g(V, V)=\lambda^{2 n-4}$. We call $V$ the fundamental (vertical) vector field.

2) If $\left(M^{4}, g\right)$ is as in Theorem 1.1 (respectively, Theorem 1.2, Theorem 1.3) and $N^{3}$ is an open subset of $\mathbb{R}^{3}$ (respectively, a constant curvature three-manifold, $\left.S^{3}\right)$, then the canonical projection $\varphi:\left(M^{4}, g\right) \rightarrow\left(N^{3}, h\right)$ is a harmonic morphism. Furthermore, if $g$ is given by the Gibbons-Hawking construction then, obviously, its fibres are generated by a Killing vector field, whilst if $g$ is given by Theorem 1.3, with $A \neq 0$, then neither is $\varphi$ horizontally homothetic nor do its fibres form a Riemannian foliation.

3) Recall [1] that any harmonic morphism with one-dimensional fibres from a Riemannian manifold of dimension at least five is submersive, whilst if the domain has dimension four then the set of critical points is discrete.

3. Characterisations of the construction and some related results. From the previous section it follows that classifying Einstein metrics which can be locally written in the form (2.1) is the same as classifying harmonic morphisms with one-dimensional fibres from Einstein manifolds. Therefore the results of this section will be given in the language of harmonic morphisms. The reader who is not primarily interested in harmonic morphisms can easily rewrite all these results in the language of Section 1.

Proposition 3.1. Let $\left(M^{4}, g\right)$ be an Einstein four-manifold and $\varphi:\left(M^{4}, g\right) \rightarrow$ $\left(N^{3}, h\right)$ a submersive harmonic morphism to a Riemannian three-manifold.

Then, the following assertions are equivalent:

(i) $\left(N^{3}, h\right)$ has constant curvature;

(ii) the following equation holds:

$$
\mathrm{d}^{\mathcal{H}}\left(\lambda^{-2}\right)=*_{\mathcal{H}} \Omega
$$

here $\mathrm{d}^{\mathcal{H}}$ is the differential followed by the orthogonal projection onto $\mathcal{H}, \lambda$ is the dilation of $\varphi, *_{\mathcal{H}}$ is the Hodge star operator on $\left(\mathcal{H}, \varphi^{*}(h)\right)$ with respect to a suitably chosen orientation, and $\Omega$ is the curvature form of the horizontal distribution (i.e., in the notation of Theorem 2.6, $\Omega=\mathrm{d} \theta$ ).

Proof. The following formula is a consequence of [26, (B.0.23)]

$$
\begin{aligned}
{ }^{M_{\text {Ricci }} \mid \mathcal{H} \otimes \mathcal{H}}=\varphi^{*} & \left.{ }^{N} \text { Ricci }\right)-\lambda^{-2}\left(\Delta^{M}(\log \lambda)+\frac{1}{4} \lambda^{6}|\Omega|_{h}^{2}\right) \varphi^{*}(h) \\
& +\frac{1}{2} \lambda^{4}\left(*_{\mathcal{H}} \Omega\right) \otimes\left(*_{\mathcal{H}} \Omega\right)-2 \mathrm{~d}^{\mathcal{H}}(\log \lambda) \otimes \mathrm{d}^{\mathcal{H}}(\log \lambda) .
\end{aligned}
$$


Assume (ii). Then from (3.2) and a Schur-type lemma (see [5] ) it easily follows that $\left(N^{3}, h\right)$ is Einstein and hence of constant curvature.

Conversely, suppose that (i) holds. Recall (see [5, Theorem 5.26]) that any Einstein manifold can be given a real-analytic structure. Then, as in [30, Proposition $1.4]$, it follows that all the objects appearing in (3.2) are real-analytic.

From (3.2) it follows that $\Omega=0$ if and only if $\mathrm{d}^{\mathcal{H}} \lambda=0$. Otherwise, by realanalyticity, both are non-zero on a dense open subset of $M^{4}$ and we may choose a real-analytic positive orthonormal local frame $\left\{X_{1}, X_{2}, X_{3}\right\}$ for $\left(\mathcal{H}, \varphi^{*}(h)\right)$ such that $X_{i}(\lambda)$ is nowhere zero for each $i=1,2,3$. From (3.2) it follows that

$$
\left(*_{\mathcal{H}} \Omega\right)\left(X_{i}\right)\left(*_{\mathcal{H}} \Omega\right)\left(X_{j}\right)=X_{i}\left(\lambda^{-2}\right) X_{j}\left(\lambda^{-2}\right)
$$

for any $i, j=1,2,3, i \neq j$. Equation (3.1) follows easily from (3.3).

Next, we give the proof of Theorem 1.5.

Proof. [Proof of Theorem 1.5] Part (a) follows from the main result of [27] (see [26, Corollary 3.4.5], and the proof of [30, Theorem 2.8]), by noting that Theorem 1.5 can be viewed as a classification result for harmonic morphisms with one-dimensional fibres on Einstein four-manifolds.

Part (b) is a consequence of Proposition 3.1. Indeed, suppose that both $\left(M^{4}, g\right)$ and $\left(N^{3}, h\right)$ are Einstein. If $\Omega=0$ on $M$ then, from (3.1), it follows that $\lambda$ is constant along horizontal curves, and Proposition 2.4 implies that, locally, $g$ is a warped product. Then, (1.3) (with $n=3$ ) follows, for example, from [5, 9.109] (see also [26]), and hence assertion (ii) of Theorem 1.5 holds.

If $\Omega \neq 0$, then, as in the previous proof, $\Omega$ is real-analytic; hence it is non-zero on a dense open subset of $M^{4}$. Now, note that the right hand side of (3.1) is basic. Hence $V\left(X\left(\lambda^{-2}\right)\right)=0$ for any basic vector field $X \in \Gamma(\mathcal{H})$ where $V$ is the fundamental vector field (Remark 2.7(1)). But $V$ commutes with basic vector fields (because $\mathcal{H}$ is a local principal connection on $\varphi$ ), and hence $V\left(\lambda^{-2}\right)$ is constant along horizontal curves. It follows that, if $V\left(\lambda^{-2}\right)$ is non-constant then $\mathcal{H}$ is integrable, equivalently, $\Omega=0$. Thus we must have that $V\left(\lambda^{-2}\right)=c$ for some constant $c \in \mathbb{R}$.

If $c=0$, then $\lambda$ is constant along the fibres of $\varphi$ (equivalently, $g$ is as in assertion (i) of Theorem 1.5), and from (3.1) it will follow that $g$ is locally given by the GibbonsHawking construction, once we have shown that $\left(N^{3}, h\right)$ is flat; this will follow from (3.6) below.

If $c \neq 0$, then $(1 / c) \mathrm{d}\left(\lambda^{-2}\right)$ is a (flat) principal connection on $\varphi$. Let $A \in \Gamma\left(T^{*} N\right)$ be a local connection form of $\mathcal{H}$ with respect to $(1 / c) \mathrm{d}\left(\lambda^{-2}\right)$, that is, $A$ is the one-form on $N$ which satisfies

$$
\theta=\frac{1}{c} \mathrm{~d}\left(\lambda^{-2}\right)+\varphi^{*}(A)
$$

From (3.1) and (3.4), it follows that

$$
-c \varphi^{*}(A)=\mathrm{d}^{\mathcal{H}}\left(\lambda^{-2}\right)=*_{\mathcal{H}} \Omega=*_{\mathcal{H}} \varphi^{*}(\mathrm{~d} A)=\varphi^{*}(* \mathrm{~d} A) .
$$

Hence $\mathrm{d} A+c * A=0$ which implies assertion (iii), except for the fact that $N^{3}$ has constant sectional curvature equal to $c^{2} / 4$ which we shall now prove.

Let $\varphi:\left(M^{4}, g\right) \rightarrow\left(N^{3}, h\right)$ be a submersive harmonic morphism between Riemannian manifolds of dimension four and three, respectively. If (3.1) is satisfied then, by applying [26, Lemma B.0.19], we get that the Ricci tensors of $(M, g)$ and $(N, h)$ 
satisfy the following relations:

$$
\begin{aligned}
& { }^{M} \text { Ricci }\left.\right|_{\mathcal{V} \otimes \mathcal{V}}=0, \quad{ }^{M} \text { Ricci }\left.\right|_{\mathcal{V} \otimes \mathcal{H}}=0, \\
& \left.{ }^{M_{\text {Ricci }}}\right|_{\mathcal{H} \otimes \mathcal{H}}=\varphi^{*}\left({ }^{\left.N_{\text {Ricci }}\right)}-\frac{c^{2}}{2} \varphi^{*}(h)\right. \text {. }
\end{aligned}
$$

From (3.6) it follows easily that, if $\left(M^{4}, g\right)$ is Einstein and (3.1) holds, then it is Ricci-flat and $\left(N^{3}, h\right)$ has constant sectional curvature equal to $c^{2} / 4$.

REMARK 3.2. The proof of Theorem 1.5 shows that (3.1) unifies the $S^{1}$-monopole equation and Beltrami fields equation. Therefore our construction may be considered to be a natural extension of the Gibbons-Hawking ansatz.

Theorem 1.3 follows from the following result.

THEOREM 3.3. Let $\varphi:\left(M^{4}, g\right) \rightarrow\left(N^{3}, h\right)$ be a surjective submersive harmonic morphism such that $\mathrm{d}^{\mathcal{H}}\left(\lambda^{-2}\right)=*_{\mathcal{H}} \Omega$ with $V\left(\lambda^{-2}\right)=c(\in \mathbb{R})$.

Then the following assertions are equivalent:

(i) $\left(M^{4}, g\right)$ is Einstein;

(ii) $\left(N^{3}, h\right)$ has constant sectional curvature equal to $c^{2} / 4$.

Moreover, if (i) or (ii) holds, then $\left(M^{4}, g\right)$ is Ricci-flat and self-dual.

Proof. The equivalence of (i) and (ii) follows from (3.6).

To establish the last statement, we may assume for simplicity that, if $c \neq 0$, $\left(N^{3}, h\right)$ is the three-sphere of radius $2 / c$ with its canonical metric and, if $c=0$, $\left(N^{3}, h\right)$ is the Euclidean three-space. Take $p$ to be the Hopf fibration $S^{3} \rightarrow S^{2}$ if $c \neq 0$, or an orthogonal projection $\mathbb{R}^{3} \rightarrow \mathbb{R}^{2}$ if $c=0$.

Then, $\psi=p \circ \varphi$ is a submersive harmonic morphism with two-dimensional fibres. Let $\mathcal{F}=\operatorname{ker} \mathrm{d} \psi$ and define $J$ to be the (negatively oriented) almost Hermitian structure on $\left(M^{4}, g\right)$ given by rotation through angle $-\pi / 2$ on $\mathcal{F}$ and rotation through angle $\pi / 2$ on $\mathcal{F}^{\perp}$.

A straightforward calculation shows that $\left(M^{4}, J, g\right)$ is a Kähler manifold if and only if $\mathrm{d}^{\mathcal{H}}\left(\lambda^{-2}\right)=*_{\mathcal{H}} \Omega$ with $V\left(\lambda^{-2}\right)=c$. It follows that $\left(M^{4}, g\right)$ is hyper-Kähler and, in particular, Ricci-flat and self-dual (see [5]). (Note that the orientation of $M^{4}$ is given as follows: orient $\mathcal{V}=\operatorname{ker} \mathrm{d} \varphi$ by $\theta$ and then choose the orientation on $M^{4}$ such that the canonical vector bundle isomorphisms $T M=\mathcal{V} \oplus \mathcal{H}$ and $\mathcal{H}=\varphi^{*}(T N)$ are orientation preserving.)

REMARK 3.4. 1) The second part of the proof of Theorem 3.3 was inspired by C. LeBrun's discussion of the Gibbons-Hawking ansatz [24] (see also [34], [9, Theorem 3.5]).

2) In [31], the method is developed to give the following:

Let $\left(N^{3}, h\right)$ be a constant curvature three-manifold and let $A$ be a real-analytic one-form on $N$. Define a Riemannian metric on $(0, \infty) \times N^{3}$ by

$$
g=\rho h+\rho^{-1}(\mathrm{~d} \rho+A)^{2} \quad(\rho \in(0, \infty)) .
$$

Then $g$ is self-dual (respectively, anti-self-dual) if and only if the following Beltrami fields equation holds on $N^{3}$ :

$$
\mathrm{d} A=-* A \quad(\text { respectively, } \mathrm{d} A=* A) .
$$

Note that $\left(N^{3}, h\right)$ may have constant sectional curvature of sign unrelated to that in (3.8) and that $g$ is not. in general, Einstein. 
We shall give details on this construction in [31], together with another new construction of self-dual metrics.

We end this section with the classification of harmonic morphisms with onedimensional fibres on compact Einstein four-manifolds. For this we need the following, which improves one of the statements of [28, Theorem 2.9].

Proposition 3.5. Let $\left(M^{4}, g\right)$ be an Einstein four-manifold and let $\varphi$ : $\left(M^{4}, g\right) \rightarrow\left(N^{3}, h\right)$ be a harmonic morphism with one-dimensional fibres to a Riemannian three-manifold.

If $\varphi$ has critical points, then $\left(M^{4}, g\right)$ is Ricci-flat and there exists a (real-analytic) Killing vector field tangent to the fibres of $\varphi$ whose zero set is equal to the set of critical points of $\varphi$.

Proof. By a result of P. Baird [1], a harmonic morphism $\varphi:(M, g) \rightarrow(N, h)$ with one-dimensional fibres is submersive if $\operatorname{dim} M>4$, whilst, if $\operatorname{dim} M=4$, the set of critical points of $\varphi$ is discrete.

Now Theorem 1.5 is equivalent to a classification result for harmonic morphisms with one-dimensional fibres on Einstein four-manifolds [27, Corollary 1.9] , [26, Corollary 3.4.5] . As already explained, if assertion (ii) of Theorem 1.5 holds, then $\varphi$ is a harmonic map which is horizontally homothetic, and hence, by a result of B. Fuglede $[14]$, it is submersive.

If assertion (iii) of Theorem 1.5 holds then $V\left(\lambda^{-2}\right)$ is a non-zero constant where, as before, $\lambda$ is the dilation of $\varphi$ and $V$ is the fundamental vector field (Remark 2.7(1)). Because the critical points of $\varphi$ are isolated, from [10] it follows that, in the neighbourhood of a critical point, $\varphi$ is topologically equivalent to the cone over the Hopf fibration $S^{3} \rightarrow S^{2}$. Therefore there exists a connected component of a fibre of $\varphi$ which is diffeomorphic to $S^{1}$, and hence at some point we must have $V\left(\lambda^{-2}\right)=0$. Thus, if (iii) of Theorem 1.5 holds, then $\varphi$ is submersive.

We have thus shown that, if $\varphi$ has critical points, then its fibres are generated by a Killing vector field, namely $V$, which can be extended to a real-analytic Killing vector field on $\left(M^{4}, g\right)$ by setting it equal to zero at the critical points (see the proof of [27, Corollary 3.3] or [26, Corollary 3.6.3]).

Because $\lambda$ and $\Omega$ are basic they are locally the pull-backs of a function and a two-form, respectively, which are defined on $N^{3}$. For simplicity, we shall denote the corresponding objects on $N^{3}$ by the same letters $\lambda$ and $\Omega$. Recall that ${ }^{M}$ Ricci $=c^{M} g$ and $\left.g\right|_{\mathcal{H}}=\lambda^{-2} \varphi^{*}(h)$. Thus (3.2) can be written as an equation on $N^{3}$. Furthermore, by applying [26, (B.0.25)], the corresponding equation on $N^{3}$ can be written as follows:

$$
{ }^{N} \text { Ricci }=2 c^{M} \lambda^{-2} h-\frac{1}{2} \lambda^{4}(* \Omega) \otimes(* \Omega)+2 \lambda^{-2} \mathrm{~d} \lambda \otimes \mathrm{d} \lambda
$$

From (3.9), it follows that any vector orthogonal to both $(* \Omega)^{\sharp}$ and $\operatorname{grad} \lambda$ is an

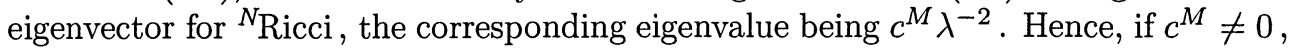
this eigenvalue tends to $\infty$ as we approach a critical value of $\varphi$, which is obviously impossible (apply, for example, [30, Lemma 2.1]). Thus $c^{M}=0$, i.e., ${ }^{M}$ Ricci $=0$.

By applying Proposition 3.5 we obtain the following result, which improves $[27$, Theorem 3.8, Theorem 4.11].

THEOREM 3.6. Let $\left(M^{4}, g\right)$ be a compact Einstein four-manifold and $\varphi$ : $\left(M^{4}, g\right) \rightarrow\left(N^{3}, h\right)$ a non-constant harmonic morphism to a Riemannian threemanifold. 
Then, up to homotheties and Riemannian coverings, $\varphi$ is the canonical projection $T^{4} \rightarrow T^{3}$ between flat tori.

Proof. If $\varphi$ is submersive this follows from [27, Theorem 3.8].

If $\varphi$ has critical points then, by Proposition $3.5,\left(M^{4}, g\right)$ is Ricci-flat and there exists a Killing vector field $V$ tangent to the fibres of $\varphi$ whose zero set is equal to the set of critical points of $\varphi$. But, since $\left(M^{4}, g\right)$ is compact and Ricci-flat, by a well-known result of S. Bochner (see $[5,1.84]$ ) $V$ is actually parallel and hence of constant norm. It follows that $\varphi$ must be submersive and the theorem is proved.

4. Solving $\mathrm{d} A+2 * A=0$ on $S^{3}$. In what follows we regard $S^{3}$ as the Lie group $\mathrm{Sp}(1)$ of unit quaternions. Let $\left\{X_{j}\right\}$ be an orthonormal positively oriented left invariant frame on $S^{3},\left\{\theta_{j}\right\}$ its dual and $\pi: S^{3} \rightarrow S^{2}\left(\frac{1}{2}\right)$ the Hopf fibration chosen so that its fibres are tangent to $X_{3}$; here $S^{2}\left(\frac{1}{2}\right)$ denotes the sphere of radius $1 / 2$. For any three-dimensional submanifold (with boundary) $N \subseteq S^{3}$ we shall denote the space of harmonic function on $N$ by $\mathcal{H}(N)$, the space of harmonic functions on $N$ which are basic with respect to $\pi$ by $\mathcal{H}_{b}(N)$, and the space of harmonic one-forms on $N$ by $\mathcal{H}^{1}(N)$.

Proposition 4.1. There exists a subbase $\mathcal{U}$ for the topology of $S^{3}$ such that, for each $U \in \mathcal{U}$, the closure $\bar{U}$ is a three-dimensional submanifold with boundary, and we have an isomorphism

$$
\left\{A \in \Gamma\left(T^{*} \bar{U}\right) \mid \mathrm{d} A+2 * A=0 \text { on } \bar{U}\right\} \stackrel{\sim}{\longrightarrow} \frac{\mathcal{H}(\bar{U}) \times \mathcal{H}(\bar{U}) \times \mathcal{H}_{b}(\bar{U})}{\mathcal{H}^{1}(\bar{U})}
$$

given by

$$
A=A_{j} \theta^{j} \longmapsto\left(A_{1}, A_{2}, A_{3}+\psi_{\bar{U}}\left(X_{1}\left(A_{1}\right)+X_{2}\left(A_{2}\right)\right)\right) \quad\left(\bmod \mathcal{H}^{1}(\bar{U})\right)
$$

where $\psi_{\bar{U}}: \mathcal{H}(\bar{U}) \rightarrow \mathcal{H}(\bar{U})$ is a certain injective linear map such that $X_{3}\left(\psi_{\bar{U}}(u)\right)=u$ for any $u \in \mathcal{H}(\bar{U})$.

Proof. Firstly, we construct the subbase $\mathcal{U}$ and the functor $\psi$. For each closed 2-ball $D$ in $S^{2}$ let $s: D \rightarrow S^{3}$ be a section of $\pi$. Because $\pi^{-1}(D)$ is a solid torus we can find an open 3-ball $U$ whose closure $\bar{U} \subseteq \pi^{-1}(D)$ is a closed 3-ball such that $s(D) \subseteq \bar{U}$ and $\left.\pi\right|_{\bar{U}}$ has connected fibres. We define $\mathcal{U}$ to be formed of all such $U$.

Let $U \in \mathcal{U}$. To define $\psi_{\bar{U}}$ let $u \in \mathcal{H}(\bar{U})$ and take $w: \bar{U} \rightarrow \mathbb{R}$ to be the smooth function characterised by

$$
X_{3}(w)=u,\left.\quad w\right|_{s(\pi(\bar{U}))}=0 .
$$

Because $X_{3}$ is a Killing vector field, we have

$$
X_{3}(\Delta w)=\Delta\left(X_{3}(w)\right)=\Delta u=0 .
$$

Hence we can find $\widetilde{w}: \pi(\bar{U}) \rightarrow \mathbb{R}$ such that

$$
\pi^{*}(\widetilde{w})=\Delta w
$$

Take $w_{1}: \pi(\bar{U}) \rightarrow \mathbb{R}$ to be the unique solution of the following Dirichlet problem:

$$
\Delta^{S^{2}} w_{1}=\widetilde{w},\left.\quad w_{1}\right|_{\partial \pi(\bar{U})}=0
$$


Define $\psi_{\bar{U}}(u)=w-\pi^{*}\left(w_{1}\right)$; we show that $X_{3}\left(\psi_{\bar{U}}(u)\right)=u$ and $\Delta\left(\psi_{\bar{U}}(u)\right)=0$. Indeed, by (4.2), we have

$$
X_{3}\left(\psi_{\bar{U}}(u)\right)=X_{3}(w)-X_{3}\left(\pi^{*}\left(w_{1}\right)\right)=u-0=u .
$$

Also, by (4.3) and (4.4), and since $\pi$ intertwines Laplacians (see, for example, [4, Chapter 4]) we have

$$
\begin{aligned}
\Delta\left(\psi_{\bar{U}}(u)\right) & =\Delta w-\Delta\left(\pi^{*}\left(w_{1}\right)\right) \\
& =\pi^{*}(\widetilde{w})-\pi^{*}\left(\Delta^{S^{2}} w_{1}\right) \\
& =\pi^{*}\left(\widetilde{w}-\Delta^{S^{2}} w_{1}\right)=0 .
\end{aligned}
$$

For a one-form $A=A_{j} \theta^{j}$ on $S^{3}$ set $\Delta A=\left(\Delta A_{j}\right) \theta^{j}$. (Note that $\Delta$ is not the Hodge Laplacian $\mathrm{d}^{*} \mathrm{~d}+\mathrm{d}^{*}$.) To prove that (4.1) is an isomorphism, it is sufficient to prove the following two facts:

(i) The map $\mathcal{H}(\bar{U}) \times \mathcal{H}(\bar{U}) \times \mathcal{H}_{b}(\bar{U}) \longrightarrow\left\{A \mid \Delta A=0, \mathrm{~d}^{*} A=0\right\}$ given by

$$
\left(u_{1}, u_{2}, u_{3}\right) \longmapsto\left(u_{1}, u_{2}, u_{3}-\psi_{\bar{U}}\left(X_{1}\left(u_{1}\right)+X_{2}\left(u_{2}\right)\right)\right) \text { is an isomorphism; }
$$

(ii) $\left\{A \mid \Delta A=0, \mathrm{~d}^{*} A=0\right\}=\left\{A \mid\left((* \mathrm{~d})^{2}+2(* \mathrm{~d})\right)(A)=0, \mathrm{~d}^{*} A=0\right\}$.

The proof of (i) follows easily from the properties of $\psi_{\bar{U}}$ and the fact that $\mathrm{d}^{*} A=$ $-\left(X_{1}\left(A_{1}\right)+X_{2}\left(A_{2}\right)+X_{3}\left(A_{3}\right)\right)$.

To prove (ii) we use the Dirac operator D : $\Gamma\left(S^{3} \times \mathbb{H}\right) \rightarrow \Gamma\left(S^{3} \times \mathbb{H}\right)$ defined by

$$
\mathrm{D}=\left(\begin{array}{cccc}
0 & -X_{1} & -X_{2} & -X_{3} \\
X_{1} & 0 & -X_{3} & X_{2} \\
X_{2} & X_{3} & 0 & -X_{1} \\
X_{3} & -X_{2} & X_{1} & 0
\end{array}\right)
$$

Then, if $A=\operatorname{Re} A+\operatorname{Im} A: S^{3} \rightarrow \mathbb{H}$ we have

$$
\mathrm{D} A=\mathrm{d}^{*}(\operatorname{Im} A)+(\mathrm{d}(\operatorname{Re} A)+(* \mathrm{~d}+2)(\operatorname{Im} A)) .
$$

Also, $\mathrm{D}^{2}=\Delta+2 \mathrm{D}$ and the proof follows.

REMARK 4.2. Proposition 4.1 and its proof are similar to the discussion of the Beltrami fields equation on $\mathbb{R}^{3}$ in [20]. This discussion admits the following reformulation (which we do not imagine to be new):

Let $\Delta$ be the Hodge Laplacian on an oriented Riemannian three-manifold $N^{3}$. Then for a coclosed one-form $A$ the vector wave equation $\Delta A=c^{2} A$, where $c$ is a constant, reads

$$
(* \mathrm{~d}+c)(* \mathrm{~d}-c)(A)=0 \text {. }
$$

Therefore we have a linear map from the space of coclosed solutions of the vector wave equation to the space of solutions of the Beltrami fields equation

$$
* \mathrm{~d} A=c A
$$

given by

$$
A \longmapsto(* \mathrm{~d}+c)(A)
$$


which is easily seen to be surjective if $c \neq 0$.

The following result is an immediate consequence of Proposition 4.1 (see [26] or [27] for a different argument).

COROLlary 4.3. The set of global solutions to $\mathrm{d} A+2 * A=0$ on $S^{3}$ is equal to the space of left invariant one-forms.

EXAMPLE 4.4. If $A$ is a left-invariant one-form on $\left(S^{3}, h\right)$ considered with its canonical metric, then $g=\rho^{2} h+\rho^{-2}(\rho \mathrm{d} \rho+A)^{2}$ is the Eguchi-Hanson metric II [12].

\section{REFERENCES}

[1] P. BAIRD, Harmonic morphisms and circle actions on 3- and 4-manifolds, Ann. Inst. Fourier (Grenoble), 40 (1990), pp. 177-212.

[2] P. BAIRd, J. Eells, A conservation law for harmonic maps, Geometry Symposium. Utrecht 1980, pp. 1-25, Lecture Notes in Math. 894, Springer-Verlag, Berlin, Heidelberg, New York, 1981.

[3] P. BAIRD, J.C. Wood, Harmonic morphisms, Seifert fibre spaces and conformal foliations, Proc. London Math. Soc., 64 (1992), pp. 170-196.

[4] P. BAIRD, J.C. WooD, Harmonic morphisms between Riemannian manifolds, London Math. Soc. Monogr. (N.S.), Oxford Univ. Press (to appear).

[5] A.L. BESSE, Einstein manifolds, Ergebnisse der Mathematik und ihrer Grenzgebiete (3), 10, Springer-Verlag, Berlin-New York, 1987.

[6] J.P. Bourguignon, A mathematician's visit to Kaluza-Klein theory, Conference on Partial Differential Equations and Geometry (Torino, 1988). Rend. Sem. Mat. Univ. Politec. Torino 1989, Special Issue, (1990), pp. 143-163.

[7] R.L. BRYANT, Harmonic morphisms with fibers of dimension one, Comm. Anal. Geom., 8 (2000), pp. 219-265.

[8] D.M.J. Calderbank, Selfdual Einstein metrics and conformal submersions, preprint, Edinburgh University, 2000 (http://xxx.arXiv.org/abs/math.DG/0001041).

[9] Q.Y. Cheng, Y.X. Dong, Some notes on harmonic morphisms, Kexue Tongbao (Chinese), 41 (1996), pp. 1825-1828.

[10] P.T. Church, J.G. Timourian, Maps with 0-dimensional critical set, Pacific J. Math., 57 (1975), pp. 59-66.

[11] J. Eells, L. Lemaire, Selected topics in harmonic maps, CBMS Regional Conference Series in Mathematics, 50, Published for the Conference Board of the Mathematical Sciences, Washington, DC; by the American Mathematical Society, Providence, RI, 1983.

[12] T. Eguchi, A.J. Hanson, Asymptotically flat self-dual solutions to Euclidean gravity, Phys. Lett. B, 74 (1978), pp. 249-251.

[13] B. Fuglede, Harmonic morphisms between Riemannian manifolds, Ann. Inst. Fourier (Grenoble), 28 (1978), pp. 107-144.

[14] B. FUGLEDE, A criterion of non-vanishing differential of a smooth map, Bull. London Math. Soc., 14 (1982), pp. 98-102.

[15] G.W. Gibbons, S.W. HaWking, Gravitational multi-instantons, Phys. Lett. B, 78 (1978), pp. 430-432.

[16] S. Gudmundsson, The Geometry of Harmonic Morphisms, Ph.D. thesis, University of Leeds, 1992.

[17] S. Gudmundsson, The Bibliography of Harmonic Morphisms, http://www.maths.lth.se/matematiklu/personal/sigma/harmonic/bibliography.html

[18] S.W. Hawking, Gravitational Instantons, Phys. Lett. A, 60 (1977), pp. 81-83.

[19] T. IshIHARA, A mapping of Riemannian manifolds which preserves harmonic functions, J. Math. Kyoto Univ., 19 (1979), pp. 215-229.

[20] P.C. Kendall, C. Plumpton, Magnetohydrodynamics, with Hydrodynamics, Pergamon Press, Oxford, New-York, Macmillan, 1964.

[21] S. KoBAYASHI, Transformation groups in differential geometry, reprint of the 1972 edition, Classics in Mathematics, Springer-Verlag, Berlin, 1995.

[22] S. KoвAYAshi, K. Nomizu, Foundations of differential geometry, I, II, Interscience Tracts in Pure and Applied Math. 15, Interscience Publ., New York, London, Sydney, 1963, 1969.

[23] H.B. Lawson, JR., M.L. Michelsohn, Spin geometry, Princeton Mathematical Series, 38, Princeton University Press, Princeton, NJ, 1989. 
[24] C. LeBrun, Complete Ricci-flat Kähler metrics on $\mathbb{C}^{n}$ need not be flat, Several complex variables and complex geometry, Part 2 (Santa Cruz, CA, 1989), pp. 297-304, Proc. Sympos. Pure Math., 52, Part 2, Amer. Math. Soc., Providence, RI, 1991.

[25] R. PAntilie, Harmonic morphisms with one-dimensional fibres, Internat. J. Math., 10 (1999), pp. 457-501.

[26] R. PAntilie, Submersive harmonic maps and morphisms, Ph.D. Thesis, University of Leeds, 2000.

[27] R. PAntilie, Harmonic morphisms with 1-dimensional fibres on 4-dimensional Einstein manifolds, Comm. Anal. Geom., (to appear).

[28] R. PAntilie, J.C. Wood, New results on harmonic morphisms with one-dimensional fibres, Bull. Math. Soc. Sci. Math. Roumanie, 43 (2000), Volume in the memory of G. Vrănceanu, pp. 355-365.

[29] R. PANTILIE, J.C. Wood, Topological restrictions for circle actions and harmonic morphisms, preprint, University of Leeds, 2000, (available from http://www.amsta.leeds.ac.uk/Pure/staff/wood/wood.html).

[30] R. Pantilie, J.C. Wood, Harmonic morphisms with one-dimensional fibres on Einstein manifolds, Trans. Amer. Math. Soc., (to appear).

[31] R. PANTILIE, J.C. Wood, Harmonic morphisms with one-dimensional fibres on self-dual manifolds (in preparation).

[32] B.L. REINHART, Differential geometry of foliations. The fundamental integrability problem, Ergebnisse der Mathematik und Ihrer Grenzgebiete, 99, Springer-Verlag, Berlin-New York, 1983.

[33] J.C. Wood, Harmonic morphisms, foliations and Gauss maps, Complex differential geometry and non-linear differential equations, 145-183, Contemp. Math., 49, Amer Math. Soc., Providence, RI, 1986.

[34] J.C. WooD, Harmonic morphisms and Hermitian structures on Einstein 4-manifolds, Internat. J. Math., 3 (1992), pp. 415-439. 